\title{
Omental fibromatosis treated by laparoscopic wide surgical resection
}

\author{
David Martin ${ }^{1,2, *}$, Mirza Muradbegovic ${ }^{1}$, Snezana Andrejevic-Blant ${ }^{3}$, David Petermann ${ }^{1}$, \\ Luca Di Mare ${ }^{1,2}$
}

${ }^{1}$ Department of Visceral Surgery, University Hospital CHUV, Lausanne, Switzerland;

${ }^{2}$ Department of General and Visceral Surgery, EHC Hospital, Morges, Switzerland;

${ }^{3}$ Unilabs Pathology, Cypa-Epalinges, Lausanne, Switzerland.

\begin{abstract}
Summary The current report presents a case of an omental fibromatosis discovered incidentally in a 46-year-old woman with no particular medical history and few symptoms. A surgical biopsy was performed initially, and microscopic examination revealed myofibroblastic proliferation. After additional immunohistochemical and molecular analyses, omental fibromatosis was diagnosed. Omental fibromatosis, also called intra-abdominal desmoid, is a rare and benign tumour but can be locally aggressive. Majority of cases are asymptomatic, and difficult to diagnose based on clinical presentation and radiological investigation. Final diagnosis is usually made on histopathology and immunohistochemistry studies. Surgical wide excision is currently the treatment of choice.
\end{abstract}

Keywords: Omental fibromatosis, desmoid tumor, surgical resection

\section{Introduction}

Most intra-abdominal fibromatosis affect small bowel mesentery (1). However, the omentum, transverse or sigmoid mesocolon, or even ligamentum teres may also be affected $(2,3)$. Omental fibromatosis is a rare benign fibroproliferative process but locally aggressive which may infiltrate the adjacent organs or recur, but do not create distant metastatic lesions (2-4). The term "fibromatosis" was first described by a team of American pathologists in the early 1960s (5). Omental fibromatosis is also termed as intra-abdominal desmoids. These tumors occur in a wide age range of patients, and have no gender or race predilection (3). They are typically seen in the third and fourth decades of life and the estimated incidence is 3.7 new cases per million people per year $(6,7)$. Final diagnosis is usually made on histopathology and immunohistochemistry studies. Surgical wide excision is currently the treatment of choice (8).

Released online in J-STAGE as advance publication February 26, 2018.

*Address correspondence to:

Dr David Martin, Department of Visceral Surgery, University Hospital CHUV, Lausanne, Switzerland.

E-mail: david.martin@chuv.ch
The current report presents a case of an omental fibromatosis discovered incidentally in a 46-year-old woman with no particular medical history and few symptoms. The Regional Committee for Medical and Health Research Ethics did not require ethical approval for reporting individual cases. Written informed consent was obtained from the patient for the publication of this case report and its accompanying images.

\section{Case Report}

A 46-year-old woman, with no medical and surgical history, presented electively for an epigastric hernia cure. Due to chronic diffuse abdominal pain, increased fatigue and weight loss of $2 \mathrm{~kg}$ in a few months, it was decided to perform a laparoscopic hernia repair with concomitant exploration of the abdominal cavity.

After introduction of the optical trocar in the left flank, exploration revealed greyish-white diffuse nodules of the great omentum, as well as a more localized tumor near the right colonic angle (Figure 1). No mesenteric adenopathy, ascites, or peritoneal implants were identified. The rest of the laparoscopic exploration revealed nothing more. A surgical biopsy was performed. It was decided to stop the procedure in order to obtain anatomopathological results and to provide an 


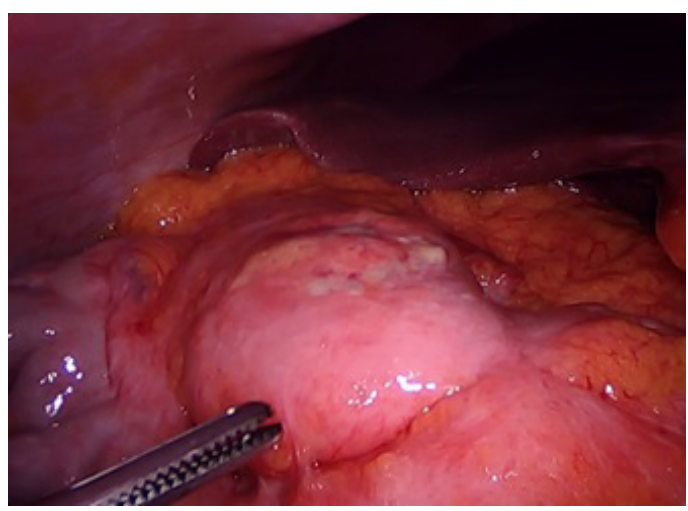

Figure 1. Exploratory laparoscopy: greyish-white nodule of the great omentum.

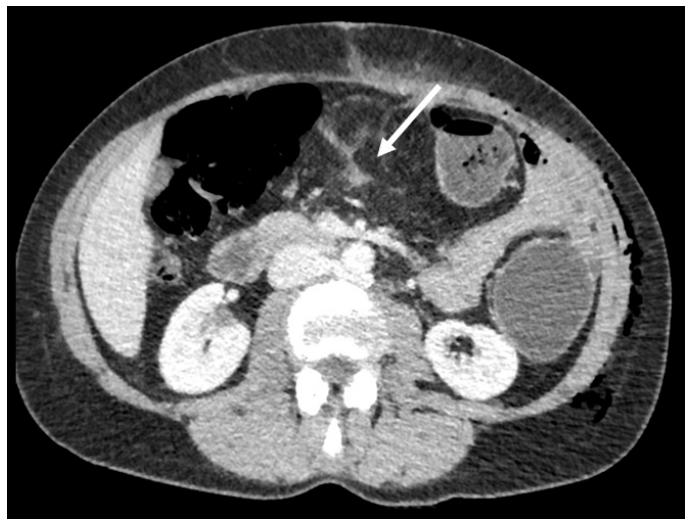

Figure 2. Abdominal CT scan showing a discrete fat infiltration of the greater omentum (white arrow), located above the transverse colon, without individualizable nodule.

oncological assessment.

The retrospective clinical examination was featureless. Tumor markers (CEA, CA 19-9, CA 125) were within the normal range. Thoraco-abdominal CT scan showed only a discrete fat infiltration of the greater omentum, located above the transverse colon, without individualizable nodule (Figure 2).

Macroscopic pathologic examination showed no significant lesions, except for fibrous remodelling (Figure 3a). Microscopic examination revealed adipose tissue with a clearly defined myofibroblastic fusocellular proliferation foci (Figure $3 \mathrm{a}, \mathrm{b}$, and c). After detailed immunohistochemical analysis, fusiform cells expressed smooth muscle actin (SMA), muscle specific actin (MSA) and beta-catenin (Figure 3d), and were negative for S-100 protein and CD-117. The MIB-1 cell proliferation index was very low $(<1 \%)$. This myofibroblastic proliferation was morphologically and immunohistochemically consistent with omental fibromatosis. The diagnosis of omental fibromatosis was confirmed by molecular analysis showing that exon 3 of the CTNNB1 gene was present.

After discussion at the local tumor board, it was decided to proceed to a laparoscopic en bloc resection of the greater omentum. Histopathological examination
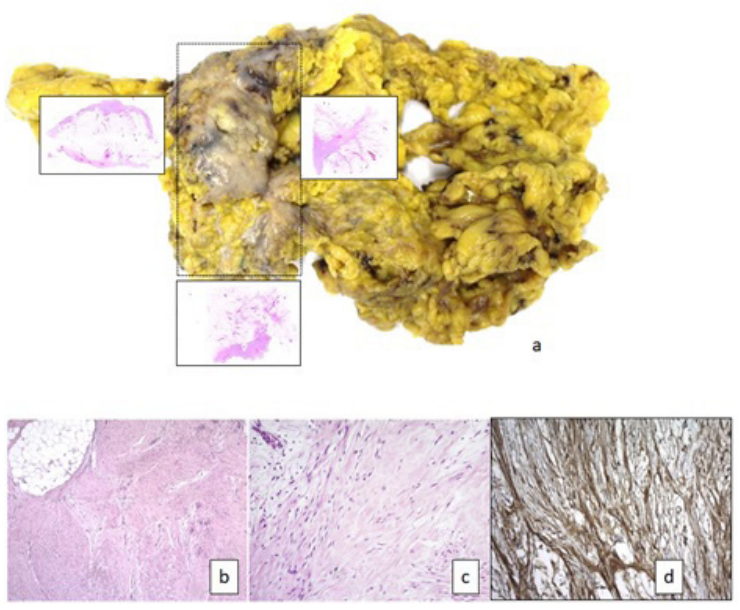

Figure 3. Macroscopic examination showing focal, fibrous remodelling (a). Microscopic examination revealing adipose tissue with a clearly defined myofibroblastic fusocellular proliferation foci (a, b, and c), expressing smooth muscle actin and beta-catenin (d).

confirmed the lesion as omental fibromatosis.

\section{Discussion}

Omental fibromatosis is a rare and benign disease occurring in a wide age range of patients, and has no gender or race predilection. Patients have few symptoms and its discovery can sometimes be unexpected during radiological imaging or intraoperatively as in this present case.

Fibromatosis have distinct biological behavior, characterized by initial rapid growth, followed by stability or even regression. Majority of fibromatosis occur sporadically, or in association with Gardener syndrome, familial adenomatosis polyposis coli (FAP) and bilateral ovarian fibromatosis $(2,9)$. Both males and females are affected with equal frequency based on literature review (5). These tumors are not encapsulated and vary widely in size (8). Most tumors measure between 5 and $10 \mathrm{~cm}$ in diameter, but they may be as large as $30 \mathrm{~cm}$ (10). Predisposing factors are pregnancy, previous abdominal surgery or trauma, and estrogen therapy $(1,11-13)$.

Patients have few symptoms until the tumor reaches a size large enough to cause pain, abdominal fullness, pressure sensation, constipation, and even weight loss $(5,8)$. Most patients with omental fibromatosis are asymptomatic or may present vague abdominal pain, as in this case (2). Some complications that have been reported include small-bowel obstruction and hydronephrosis from ureteric obstruction (14).

The imaging appearance of these tumors is variable and depends on fibroblastic proliferation, fibrosis, collagen content, and vascularity (1). Ultrasonography or computed tomography may be helpful in diagnosis but ultimately, final diagnosis is made by excision and histological examination (2). On ultrasonography, 
desmoid tumors have variable echogenicity, appearing as masses of low, medium, or high echogenicity with smooth defined margins (7). Computed tomography images typically demonstrate tumors as solid, wellcircumscribed masses of soft tissue density that do not contain calcium, with homogenous enhancement at the periphery and cystic pattern in the central region $(8,15)$.

On macroscopic examination, desmoid tumors are usually circumscribed lesions, but they may have irregular or infiltrating borders, as in this current case. On the surface, they are white and coarsely trabeculated, resembling scar tissue (1). In $10-15 \%$ of cases, they are multiple (1). Histologically, desmoid tumors are lesions composed of bland spindled or stellate fibroblastic cells embedded in a collagenous stroma, without evidence of muscular or neural differentiation and with little or no inflammatory component (1). Differential diagnosis includes cysts, sclerosing mesenteritis, mesenteric panniculitis, or tumours originating from smooth muscle, neuronal tissue, adipose and stromal tissue of gastrointestinal tract (2).

Treatment of choice is surgical resection (8). Radiation therapy and/or chemotherapy demonstrated no benefit (16). Surgical approach includes wide local excision of these tumours as they have tendency toward local recurrence in up to $50 \%(2,8)$. Some authors recommend a trial of observation with antioestrogens, nonsteroidal anti-inflammatory drugs with limited role of targeted agents such as Imatinib $(2,6)$. There are no clear guidelines for the treatment and follow-up of omental fibromatosis because of its rarity. Therefore, close longterm follow-up should be performed.

In conclusion, omental fibromatosis is usually a benign tumour but can be locally aggressive. Majority of cases are asymptomatic, and difficult to diagnose based on clinical presentation and radiological investigation. Final diagnosis is usually made on histopathology and immunohistochemistry studies. Currently, surgical excision is the only curative method of treatment.

\section{References}

1. Faria SC, Iyer RB, Rashid A, Ellis L, Whitman GJ. Desmoid tumor of the small bowel and the mesentery.
AJR Am J Roentgenol. 2004; 183:118.

2. Kori C, Singh P, Jain N, Jain J, Kumar V. Giant omental fibromatosis presenting as pelvic mass. J Clin Diagn Res. 2015; 9:XD06-XD08.

3. Misiak P, Piskorz L, Wcislo S, Jablonski S, Brocki M. Giant mesentery fibromatosis presenting as acute abdomen - Case report. Contemp Oncol (Pozn). 2013; 17:468-469.

4. Osawa H, Nishimura J, Inoue A, et al. A case of solitary fibrous tumor from the greater omentum resected via laparoscopic surgery. Gan To Kagaku Ryoho. 2014; 41:2493-2495. (in Japanese)

5. Yannopoulos K, Stout AP. Primary solid tumors of the mesentery. Cancer. 1963; 16:914-927.

6. Einstein DM, Tagliabue JR, Desai RK. Abdominal desmoids: CT findings in 25 patients. AJR Am J Roentgenol. 1991; 157:275-279.

7. Casillas J, Sais GJ, Greve JL, Iparraguirre MC, Morillo G. Imaging of intra- and extraabdominal desmoid tumors. Radiographics. 1991; 11:959-968.

8. Disher AC, Biswas M, Miller TQ, Kuvhenguhwa A. Atypical desmoid tumor of the abdomen: A case report. J Natl Med Assoc. 1993; 85:309-311.

9. Xuereb S, Xuereb R, Buhagiar C, Gauci J, Magri C. A case report of desmoid tumour-a forgotten aspect of FAP? Int J Surg Case Rep. 2017; 30:122-125.

10. Sakorafas GH, Nissotakis C, Peros G. Abdominal desmoid tumors. Surg Oncol. 2007; 16:131-142.

11. Levy AD, Rimola J, Mehrotra AK, Sobin LH. From the archives of the AFIP: benign fibrous tumors and tumorlike lesions of the mesentery: Radiologic-pathologic correlation. Radiographics. 2006; 26:245-264.

12. Gautam A, Khatri AK, Pandey M, Arya NC, Shukla VK. Mesenteric mass in a 28-year-old woman. Postgrad Med J. 1997; 73:121-122.

13. Vaswani BA, Shah M, Shah PM, Parikh BJ, Anand AS, Sharma GL. Giant mesenteric fibromatosis in Gardner's syndrome. Indian J Cancer. 2011; 48:140-142.

14. Baron RL, Lee JK. Mesenteric desmoid tumors: Sonographic and computed-tomographic appearance. Radiology. 1981; 140:777-779.

15. Paksoy Y, Sahin M, Acikgozoglu S, Odev K, Omeroglu E. Omental fibroma: CT and US findings. Eur Radiol. 1998; 8:1422-1424.

16. Schwartz RW, Reames M, McGrath PC, Letton RW, Appleby G, Kenady DE. Primary solid neoplasms of the greater omentum. Surgery. 1991; 109:543-549.

(Received December 29, 2017; Revised February 17, 2018; Accepted February 19, 2018) 